\title{
A SOBOLEV INEQUALITY AND NEUMANN HEAT KERNEL ESTIMATE FOR UNBOUNDED DOMAINS
}

\author{
Z. Q. Chen, R. J. Williams, And Z. Zhao
}

\begin{abstract}
Suppose $D$ is an unbounded domain in $\mathbb{R}^{d}(d \geq 2)$ with compact boundary and that $D$ satisfies a uniform interior cone property. We show that for $1 \leq p<d$, there exists a constant $c=c(D, p)$ such that for each $f \in W^{1, p}(D)$ the following Sobolev inequality holds:

$$
\|f\|_{q} \leq c\|\nabla f\|_{p},
$$

where $1 / q=1 / p-1 / d$ and for $r=p, q,\|\cdot\|_{r}$ denotes the norm in $L^{r}(D)$. As an application of this Sobolev inequality, assuming in addition that $D$ is a Lipschitz domain in $\mathbb{R}^{d}$ with $d \geq 3$, we obtain a Gaussian upper bound estimate for the heat kernel on $D$ with zero Neumann boundary condition.
\end{abstract}

\section{Introduction}

For a domain $U \subset \mathbb{R}^{d}$ and $p \in[1, \infty)$, we define $L^{p}(U)$ to be the space of real-valued functions defined on $U$ that are $L^{p}$-integrable relative to Lebesgue measure on $U$. The norm on $L^{p}(U)$ is given by

$$
\|f\|_{p}=\left(\int_{U}|f(x)|^{p} d x\right)^{\frac{1}{p}} .
$$

We further define

$$
W^{1, p}(U)=\left\{f \in L^{p}(U): \frac{\partial f}{\partial x_{i}} \in L^{p}(U) \text { for } i=1, \ldots, d\right\}
$$

with norm $\|f\|_{1, p} \equiv\|f\|_{p}+\|\nabla f\|_{p}$, where $\nabla f=\left(\frac{\partial f}{\partial x_{1}}, \cdots, \frac{\partial f}{\partial x_{d}}\right)$. Here the partial derivatives $\frac{\partial f}{\partial x_{i}}$ are understood in the distributional sense. Note that in the above we do not indicate the dependence of $\|\cdot\|_{p}$ and $\|\cdot\|_{1, p}$ on $U$, since usually there will only be one relevant domain $U$. If there is

1991 Mathematics Subject Classification. Primary: 46E35; Secondary: 35K05, $60 J 35$.

Key words and phrases. Sobolev inequality, cone property, imbedding theorem, Lipschitz domain, heat kernel, Neumann boundary condition.

Received October 1, 1993.

Research supported in part by NSF Grants DMS 8657483 and GER 9023335. 
any chance of ambiguity, we shall explicitly indicate the domain $U$ in the norm, for example, $\|\cdot\|_{U, p}$ for $\|\cdot\|_{p}$.

In the sequel, we assume that $D$ is an unbounded domain in $\mathbb{R}^{d}$ with compact boundary and that $D$ has the following uniform interior cone property, henceforth referred to simply as the cone property.

Cone Property. The domain $D$ is said to have the cone property if there exists a finite cone

$$
V=\left\{x=\left(x_{1}, \cdots, x_{d}\right) \in \mathbb{R}^{d}: x_{d}>\alpha\left(x_{1}^{2}+\cdots+x_{d-1}^{2}\right)^{1 / 2} \text { and }\|x\|<\beta\right\}
$$

for some $\alpha, \beta>0$ such that each point $x \in D$ is the vertex of a finite cone $V_{x}$ contained in $D$ which is congruent to $V$. Here $\|\cdot\|$ denotes the Euclidean norm on $\mathbb{R}^{d}$.

Our main result is the following.

Theorem 1. Suppose that $D$ is an unbounded domain with compact boundary and that $D$ has the cone property. Then for $1 \leq p<d$, there exists a constant $c=c(D, p)$ such that the following Sobolev inequality holds:

$$
\|f\|_{q} \leq c\|\nabla f\|_{p} \quad \text { for all } f \in W^{1, p}(D),
$$

where $1 / q=1 / p-1 / d$.

It is well known (cf. [1], [5]) that the above Sobolev inequality holds with $W_{0}^{1, p}(D)$ in place of $W^{1, p}(D)$ for arbitrary domains $D$, where $W_{0}^{1, p}(D)$ is the subspace of $W^{1, p}(D)$ obtained by completing the space of $C^{\infty}$ functions having compact support in $D$ with respect to the norm $\|\cdot\|_{1, p}$. However, the Sobolev inequality $(2)$ on $W^{1, p}(D)$ cannot hold without any restrictions on $D$. For example, (2) cannot be true for a domain $D$ with finite Lebesgue measure since in this case $1 \in W^{1, p}(D)$ and the right hand side of (2) vanishes.

In [9], using a form of capacity, Maz'ja characterizes the class $\mathcal{J}$ of open sets $D$ for which the Sobolev inequality (2) holds. He also gives the best constant $c$ in the Sobolev inequality (2) (see Theorem 4.7.4 of [9]). However we found Maz'ja's condition difficult to check in practice, despite the fact that the class $\mathcal{J}$ is closed under the operation of taking finite unions (by Theorem 4.7.4 and Proposition 4.3.1/1 in [9]). This motivated us to prove the Sobolev inequality (2) directly under the assumptions in Theorem 1. In particular, by Theorem 1 and Theorem 4 below, unbounded domains with compact boundary having the cone property and exteriors of closed convex sets are in $\mathcal{J}$.

As an application of the Sobolev inequality (2) for $p=2$ and $d \geq 3$, we shall prove Theorem 2 below. This has been applied in [3] to the study of 
semilinear elliptic equations with Neumann boundary conditions. Before we can state Theorem 2, several notions need to be introduced.

A domain $D$ is said to be Lipschitz (or $C^{0,1}$ ) if locally near $\partial D, D$ can be represented as the region lying above the graph of a Lipschitz function (see, e.g., p.244 of [5]). For such a domain $D$, denote by $\mathcal{E}$ the quadratic form defined on $W^{1,2}(D)$ by:

$$
\mathcal{E}(f, g)=\frac{1}{2} \int_{D} f(x) g(x) d x, \quad \text { for } f, g \in W^{1,2}(D) .
$$

There is a unique self-adjoint non-positive operator $\mathcal{A}$, with domain $\mathcal{D}(\mathcal{A})$, associated with $\left(W^{1,2}(D), \mathcal{E}\right)$. In particular,

$$
\begin{aligned}
\mathcal{D}(\mathcal{A})=\left\{f \in W^{1,2}(D):\right. & \exists g \in L^{2}(D) \text { s.t. } \\
& \left.\mathcal{E}(f, h)=-\int_{D} g h d x \text { for all } h \in W^{1,2}(D)\right\},
\end{aligned}
$$

and for $f$ and $g$ as in the description of $\mathcal{D}(\mathcal{A}), \mathcal{A} f=g$ (see [7]). The symmetric strongly continuous contraction semigroup $\left\{P_{t}\right\}_{t>0}$ associated with $(\mathcal{A}, \mathcal{D}(\mathcal{A}))$ has a symmetric integral kernel $p(t, x, y)$ which is smooth on $(0, \infty) \times D \times D$ and such that $P_{t} f(x)=\int_{D} p(t, x, y) f(y) d y$ a.e. on $D$ for $f \in L^{2}(D)$. See Lemma 2.11 of [6] for details on the existence of $p(t, x, y)$ (note that although in [6] domains are assumed to be bounded, the proof of the above fact works for unbounded domains as well). When $\partial D$ is smooth, $p(t, x, y)$ can be shown to be the fundamental solution for the heat equation with zero Neumann boundary condition (see [12], for example). By analogy, when $D$ is Lipschitz, we call $p(t, x, y)$ the heat kernel for $\frac{1}{2} \Delta$ on $D$ with zero Neumann boundary condition.

Theorem 2. Suppose $D$ is an unbounded domain with compact Lipschitz boundary in $\mathbb{R}^{d}$ where $d \geq 3$. Then the heat kernel $p(t, x, y)$ of $\frac{1}{2} \Delta$ on $D$ with zero Neumann boundary condition can be extended continuously to $(0, \infty) \times \bar{D} \times \bar{D} ;$ we still denote the extension by $p(t, x, y)$. Then there exist constants $c_{1}>0$ and $M>1$ such that

$$
p(t, x, y) \leq \frac{c_{1}}{t^{d / 2}} \exp \left(-\frac{|x-y|^{2}}{M t}\right), \quad \text { for all } t>0, x, y \in \bar{D} .
$$

For $x, y \in \bar{D}$, let $G(x, y)=\int_{0}^{\infty} p(t, x, y) d t$. When $G(x, y)$ is finite for all $x, y \in \bar{D}$ with $x \neq y$, it is called the Green's function for $\frac{1}{2} \Delta$ on $D$ with zero Neumann boundary condition. Integrating both sides of (3) gives: 
Corollary 3. The Green's function $G(x, y)$ for $\frac{1}{2} \Delta$ on $D$ with zero Neumann boundary condition exists and is continuous on $\bar{D} \times \bar{D}$, except on the diagonal. Furthermore, there exists a constant $c_{2}=c_{2}(D)>0$ such that

$$
G(x, y) \leq \frac{c_{2}}{|x-y|^{d-2}} \quad \text { for all } x, y \in \bar{D}
$$

\section{Proof of Theorem 1}

We begin by proving the Sobolev inequality (2) for the exterior of a closed convex set.

Theorem 4. Suppose that $U$ is the exterior of a closed convex set in $\mathbb{R}^{d}$. Then for $1 \leq p<d$ there exists a constant $c=c(U, p)$ such that for $f \in W^{1, p}(U)$,

$$
\|f\|_{q} \leq c\|\nabla f\|_{p}
$$

where $1 / q=1 / p-1 / d$. In particular, the above inequality holds for the exterior of a bounded closed ball.

Remark. In the above theorem, $U$ may have non-compact boundary.

Proof. Since $U$ is the exterior of a closed convex set, $U$ is Lipschitz (see, for example, Theorem 4.2 of $\mathrm{Ch.V}$ in [5]). Therefore by Theorem 4.7 of Chapter $\mathrm{V}$ in [5], the set of restrictions to $U$ of all $C^{\infty}$ functions with compact support in $\mathbb{R}^{d}$ is $\|\cdot\|_{1, p}$-dense in $W^{1, p}(U)$ for $p \geq 1$. Hence it suffices to prove (5) for all functions $f$ in $W^{1, p}(D)$ that are smooth in $D$ and such that $f(x)$ vanishes when $\|x\|$ is sufficiently large. Since $\mathbb{R}^{d} \backslash U$ is convex, for $x \in U$ and each $i \in\{1, \cdots, d\}$, there is a half-line in $U$ which is parallel to the $i$ th coordinate axis and has $x$ as its initial point. Thus one has, for all $x \in U$,

$$
|f(x)| \leq \int_{-\infty}^{\infty} 1_{D}(\xi)\left|\frac{\partial f}{\partial \xi_{i}}(\xi)\right| d \xi_{i}, \quad i=1,2, \ldots, d .
$$

Inequality (5) then follows from the standard argument for proving the corresponding Sobolev inequality in $W_{0}^{1, p}(D)$ (see the proof of Theorem 3.6 in Ch.V of [5], for example).

For $r>0$, denote by $B_{r}$ the ball $\left\{x \in \mathbb{R}^{d}:\|x\|<r\right\}$. Let $D_{r}=D \cap B_{r}$. Define $W_{r}^{1, p}\left(D_{r}\right)$ to be the closure in $W^{1, p}\left(D_{r}\right)$ of the set of restrictions to $D_{r}$ of all $C^{\infty}\left(\mathbb{R}^{d}\right)$ functions having compact support in $B_{r}$. Intuitively, $W_{r}^{1, p}\left(D_{r}\right)$ contains those functions in $W^{1, p}\left(D_{r}\right)$ that vanish on $\partial B_{r}$.

We have the following Poincaré inequality on $W_{r}^{1, p}\left(D_{r}\right)$ for $r>0$ such that $B_{r} \supset \partial D$. 
Lemma 5. Suppose that $1 \leq p<d$ and $r>0$ such that $B_{r} \supset \partial D$. There exists a constant $c=c(D, r, p)>0$ such that for each $f \in W_{r}^{1, p}\left(D_{r}\right)$,

$$
\|f\|_{p} \leq c\|\nabla f\|_{p}
$$

Proof. Let

$$
\lambda=\inf \left\{\frac{\|\nabla f\|_{p}}{\|f\|_{p}}: f \in W_{r}^{1, p}\left(D_{r}\right)\right\} .
$$

There exists a sequence $\left\{f_{n}\right\}_{n \geq 1} \subset W_{r}^{1, p}\left(D_{r}\right)$ such that $\left\|f_{n}\right\|_{p}=1$ for all $n$ and $\|\nabla f\|_{p}$ decreases to $\lambda$ as $n \rightarrow \infty$. Since $D_{r}$ is a bounded domain with the cone property, by the Rellich-Kondrachov compactness theorem (see [1], p.144), the imbedding $W^{1, p}\left(D_{r}\right) \hookrightarrow L^{p}\left(D_{r}\right)$ is compact for $1 \leq p<d$ (note that for this one uses the fact that $p<q \equiv p d /(d-p)$ ). Therefore, without loss of generality, we may assume that $\left\{f_{n}\right\}_{n \geq 1}$ converges in $L^{p}\left(D_{r}\right)$ to some $f$ with $\|f\|_{p}=1$. Now suppose that $\lambda=0$. Then $\left\|\nabla f_{n}\right\|_{p}$ decreases to zero as $n \rightarrow \infty$, and for any smooth function $\psi$ with compact support in $D_{r}$ and $i=1,2, \ldots, d$, using integration by parts we have

$$
\begin{aligned}
\int_{D_{r}} f(x) \frac{\partial \psi}{\partial x_{i}}(x) d x & =\lim _{n \rightarrow \infty} \int_{D_{r}} f_{n}(x) \frac{\partial \psi}{\partial x_{i}}(x) d x \\
& =-\lim _{n \rightarrow \infty} \int_{D_{r}} \frac{\partial f_{n}}{\partial x_{i}}(x) \psi(x) d x \\
& =0 .
\end{aligned}
$$

Thus $\nabla f=0$ and $f$ is a constant function on $D_{r}$. But $f$ is a limit in $W^{1, p}\left(D_{r}\right)$ of functions in $W_{r}^{1, p}\left(D_{r}\right)$ and hence $f \in W_{r}^{1, p}\left(D_{r}\right)$. The only constant function in this space is identically zero, which contradicts the fact that $\|f\|_{p}=1$. Therefore $\lambda>0$ and (7) is established with $c=1 / \lambda$.

Proof of Theorem 1. In this proof we have functions defined on different domains, $U_{1}, U_{2}, D$. To clearly indicate which domain applies for integration, for this proof only we shall use the notation

$$
\|g\|_{U, p}=\left(\int_{U}|g(x)|^{p} d x\right)^{1 / p}
$$

for a domain $U$ and $g \in L^{p}(U)$. In this proof, $c$ will denote various constants.

Let $r>1$ such that $B_{r-1} \supset \partial D$. Let $\phi$ be a $C^{\infty}$ function on $\mathbb{R}^{d}$ with compact support in $B_{r}$ such that $0 \leq \phi \leq 1$ and $\phi=1$ on $B_{r-\frac{1}{2}}$. Let $U_{1}=D \cap B_{r}$ and $U_{2}=\left\{x \in \mathbb{R}^{d}:\|x\|>r-1\right\}$. For $f \in W^{1, p}(D)$, set 
$f_{1}=f \phi$ and $f_{2}=f(1-\phi)$ on $D$. Then $f=f_{1}+f_{2}$. We next consider $f_{1}$ as an element of $W_{r}^{1, p}\left(U_{1}\right)$ and $f_{2}$ as an element of $W_{0}^{1, p}\left(U_{2}\right)$. Since $U_{1}$ is bounded and has the cone property, by the Sobolev imbedding theorem for $W^{1, p}\left(U_{1}\right)$ (cf. Theorem 5.4 of $\left.[1]\right)$,

$$
\left\|f_{1}\right\|_{U_{1}, q} \leq c\left(\left\|\nabla f_{1}\right\|_{U_{1}, p}+\left\|f_{1}\right\|_{U_{1}, p}\right)
$$

which by Lemma 5 implies

$$
\left\|f_{1}\right\|_{U_{1}, q} \leq c\left\|\nabla f_{1}\right\|_{U_{1}, p} \leq c\left(\|\nabla f\|_{D, p}+\|f \nabla \phi\|_{D, p}\right) .
$$

By Theorem 4,

$$
\left\|f_{2}\right\|_{U_{2}, q} \leq c\left\|\nabla f_{2}\right\|_{U_{2}, p} \leq c\left(\|\nabla f\|_{D, p}+\|f \nabla \phi\|_{D, p}\right) .
$$

Since $\nabla \phi$ is supported in $B_{r} \backslash B_{r-1}$, by Hölder's inequality,

$$
\|f \nabla \phi\|_{D, p}=\|f \nabla \phi\|_{U_{2}, p} \leq\|\nabla \phi\|_{D, d}\|f\|_{U_{2}, q} .
$$

Note that the restriction of $f$ to $U_{2}$ is in $W^{1, p}\left(U_{2}\right)$ and so by Theorem 4 ,

$$
\|f\|_{U_{2}, q} \leq c\|\nabla f\|_{U_{2}, p} \leq c\|\nabla f\|_{D, p} .
$$

Since $f=f_{1}+f_{2}$ where $f_{1}$ has support in $U_{1}$ and $f_{2}$ has support in $U_{2}$, combining (8)-(11) proves the Sobolev inequality (2).

\section{Proof of Theorem 2}

In this section we assume that $D$ is an unbounded domain in $\mathbb{R}^{d}$ with compact Lipschitz boundary and $d \geq 3$. Thus, the Sobolev inequality (2) yields

$$
\|f\|_{p} \leq c\|\nabla f\|_{2} \quad \text { for all } f \in W^{1,2}(D),
$$

with $p=2 d /(d-2)$. Let $p(t, x, y)$ be the heat kernel for $\frac{1}{2} \Delta$ on $D$ with zero Neumann boundary condition as described in Section 1. Recall that $p(t, x, y)$ is symmetric in $x, y$ and is smooth on $(0, \infty) \times D \times D$. By using the standard method in [4] (more specifically, Theorems 2.4.6, 2.2.3, and a straightforward adaptation of Section 3.2 to the case of zero Neumann boundary conditions), one can use (12) to show that

$$
p(t, x, y) \leq \frac{c_{3}}{t^{d / 2}} \exp \left(-\frac{|x-y|^{2}}{M t}\right), \quad \text { for all }(t, x, y) \in(0, \infty) \times D \times D,
$$

for some constants $c_{3}>0$ and $M>1$.

We are going to show now that $p(t, x, y)$ can be extended continuously to $(0, \infty) \times \bar{D} \times \bar{D}$. Let $r>0$ such that $B_{r} \supset \partial D$. Denote by $h(t, x, y)$ 
the (symmetric) heat kernel for $\frac{1}{2} \Delta$ on $D_{r}=D \cap B_{r}$ with zero Neumann boundary condition. From [2] we have that $h$ can be extended continuously to $(0, \infty) \times \bar{D}_{r} \times \bar{D}_{r}$ and that for each $T>0$ there exist constants $c_{r}=$ $c_{r}(D, r, T)>0$ and $M_{r}=M_{r}(D, r, T)>1$ such that

$$
h(t, x, y) \leq \frac{c_{r}}{t^{d / 2}} \exp \left(-\frac{|x-y|^{2}}{M_{r} t}\right) \quad \text { for all }(t, x, y) \in(0, T] \times \bar{D}_{r} \times \bar{D}_{r} .
$$

Let $\left(Y,\left\{Q_{x}, x \in \bar{D}_{r}\right\}\right)$ be the continuous strong Markov process that is (normally) reflecting Brownian motion on the bounded Lipschitz domain $D_{r}$ (see [2]). It follows from [2] that this process has $h(t, x, y)$ as its transition density function. Denote by $p_{r}(t, x, y)$ the symmetric integral kernel on $\bar{D} \cap B_{r}$ for the semigroup of $Y$ killed on hitting $\partial B_{r}$. The existence of $p_{r}$ follows from the strong Markov property of $Y$ in a similar manner to that in [10], p.33; in particular we have

$$
p_{r}(t, x, y)=h(t, x, y)-E^{Q_{x}}\left[h_{r}\left(t-\tau_{r}, Y_{\tau_{r}}, y\right) ; t>\tau_{r}\right]
$$

for $(t, x, y) \in(0, \infty) \times\left(\bar{D} \cap B_{r}\right) \times\left(\bar{D} \cap B_{r}\right)$, where $\tau_{r}=\inf \left\{t \geq 0: Y_{t} \in \partial B_{r}\right\}$. Intuitively $p_{r}(t, x, y)$ is the heat kernel for $\frac{1}{2} \Delta$ on $D_{r}$ with zero Neumann boundary condition on $\partial D$ and zero Dirichlet boundary condition on $\partial B_{r}$. For $\epsilon \in(0, r)$, by $(14)$,

$$
\begin{aligned}
|h(t, x, y)| \leq \frac{c_{r}}{t^{d / 2}} \exp \left(-\frac{\epsilon^{2}}{M_{r} t}\right) & \\
& \quad \text { for all }(t, x, y) \in(0, T] \times \partial B_{r} \times\left(\bar{D} \cap \bar{B}_{r-\epsilon}\right) .
\end{aligned}
$$

Thus as $t \rightarrow 0, h(t, x, y)$ converges to zero uniformly for $(x, y) \in \partial B_{r} \times$ $\left(\bar{D} \cap \bar{B}_{r-\epsilon}\right)$. Therefore $\{h(t, x, y)\}_{x \in \partial B_{r}}$ as a family of functions of $(t, y)$ is equi-continuous on $(0, T] \times\left(\bar{D} \cap \bar{B}_{r-\epsilon}\right)$. Since $Y_{\tau_{r}} \in \partial B_{r}$,

$$
\left\{E^{Q_{x}}\left[h\left(t-\tau_{r}, Y_{\tau_{r}}, y\right) ; t>\tau_{r}\right]\right\}_{x \in \bar{D} \cap B_{r}}
$$

is equi-continuous for $(t, y) \in(0, T] \times\left(\bar{D} \cap \bar{B}_{r-\epsilon}\right)$. It follows from (15) that $\left\{p_{r}(t, x, y)\right\}_{x \in \bar{D} \cap \bar{B}_{r-\epsilon}}$ is equi-continuous for $(t, y) \in[\epsilon, T] \times\left(\bar{D} \cap \bar{B}_{r-\epsilon}\right)$ for any $\epsilon \in(0, T)$. Since $p_{r}(t, x, y)$ is symmetric in $(x, y), p_{r}(t, x, y)$ is uniformly continuous on $[\epsilon, T] \times\left(\bar{D} \cap \bar{B}_{r-\epsilon}\right) \times\left(\bar{D} \cap \bar{B}_{r-\epsilon}\right)$. Therefore $p_{r}(t, x, y)$ is continuous on $(0, T) \times\left(\bar{D} \cap B_{r}\right) \times\left(\bar{D} \cap B_{r}\right)$ for each $T>0$.

On the other hand, if $\left(X,\left\{P_{x}, x \in \bar{D}\right\}\right)$ denotes the continuous strong Markov process that is (normally) reflecting Brownian motion on $\bar{D}$, then similar to $(15)$ we have for $(t, x, y) \in(0, \infty) \times D_{r} \times D_{r}$,

$$
p_{r}(t, x, y)=p(t, x, y)-E^{P_{x}}\left[p\left(t-\tau_{r}, X_{\tau_{r}}, y\right) ; t>\tau_{r}\right],
$$


where $\tau_{r}=\inf \left\{t \geq 0: X_{t} \in \partial B_{r}\right\}$. Since $X_{\tau_{r}} \in \partial B_{r}$, it follows from the inequality (13) that for each positive integer $k$, as a function of $(t, x, y)$ in $(0, \infty) \times D_{k} \times D_{k}, E^{P_{x}}\left[p\left(t-\tau_{r}, X_{\tau_{r}}, y\right) ; t>\tau_{r}\right]$ converges to zero uniformly as $r \rightarrow \infty$, where $D_{k}=D \cap B_{k}$. Therefore for each $k>0, p_{r}(t, x, y)$ converges to $p(t, x, y)$ uniformly on $(0, \infty) \times D_{k} \times D_{k}$ as $r \rightarrow \infty$. Hence $p(t, x, y)$ can be extended continuously to $(0, \infty) \times \bar{D}_{k} \times \bar{D}_{k}$ and therefore on $(0, \infty) \times \bar{D} \times \bar{D}$. Thus inequality (13) holds for $(t, x, y) \in(0, \infty) \times \bar{D} \times \bar{D}$. Theorem 2 is now proved.

\section{Acknowledgement}

The authors would like to thank Salah Baouendi, Carlos Kenig and Peter Li for helpful discussions and suggestions.

\section{References}

1. R. A. Adams, Sobolev Spaces. Academic Press, 1975.

2. R. F. Bass and P. Hsu, Some potential theory for reflecting Brownian motion in Hölder and Lipschitz domains. Ann. Probab. 19 (1991), 486-508.

3. Z. Q. Chen, R. J. Williams and Z. Zhao, On the existence of positive solutions for semilinear elliptic equations with Neumann boundary conditions. Preprint.

4. E. B. Davies, Heat Kernels and Spectral Theory. Cambridge University Press, Cambridge, U.K., 1989.

5. D. E. Edmunds and W. D. Evans, Spectral Theory and Differential Operators. Clarendon Press, Oxford, 1987.

6. M. Fukushima, A construction of reflecting barrier Brownian motions for bounded domains. Osaka J. Math. 4 (1967), 183-215.

7. M. Fukushima, Dirichlet Forms and Markov Processes. North-Holland, Amsterdam, 1980.

8. P. Li and S.-T. Yau, Asymptotically flat complete Kähler manifolds. In Pure and Applied Mathematics 143, edited by G. Komatsu and V. Sakane, Marcel Dekker, New York-Basel-Hong Kong, 1992, 131-143.

9. V. G. Maz'ja, Sobolev Spaces. Springer-Verlag, Berlin-Heidelberg-New York, 1985.

10. S. C. Port and C. J. Stone, Brownian Motion and Classical Potential Theory. Academic Press, New York, 1978.

11. D. W. Stroock, Diffusion semigroups corresponding to uniformly elliptic divergence form operators. In Lecture Notes in Mathematics 1321, Springer-Verlag, BerlinHeidelberg-New York, 1988, 316-347.

12. K. Sato and T. Ueno, Multi-dimensional diffusion and the Markov process on the boundary. J. Math. Kyoto Univ. 4-5 (1965), 529-605.

Department of Mathematics, University of California, San Diego, La Jolla, CA 92093-0112, USA

E-mail address: zchen@euclid.ucsd.edu, rjwilliams@ucsd.bitnet

Department of Mathematics, University of Missouri, Columbia, Missouri 65211, USA

E-mail address: mathzz@mizzou1.missouri.edu 